\title{
Downregulation of TIM-3 mRNA expression in peripheral blood mononuclear cells from patients with systemic lupus erythematosus
}

\author{
X.Z. Cai ${ }^{1,2}$, W.Y. Huang ${ }^{1}$, Y. Qiao ${ }^{1}$, Y. Chen ${ }^{1}$, S.Y. Du ${ }^{1}$, D. Chen ${ }^{1}$, S. Yu ${ }^{1}$, N. Liu ${ }^{3}$ \\ L.Y. Dou ${ }^{1}$ and Y. Jiang ${ }^{1,2,4}$ \\ ${ }^{1}$ Central Laboratory, First Affiliated Hospital, China Medical University, Shenyang, China \\ ${ }^{2}$ Department of Immunology, College of Basic Medical Sciences, China Medical University, Shenyang, China \\ ${ }^{3}$ Department of Nephrology, First Affiliated Hospital, China Medical University, Shenyang, China \\ ${ }^{4}$ Department of Dermatology, First Affiliated Hospital, China Medical University, Shenyang, China
}

\begin{abstract}
The T-cell immunoglobulin and mucin domain (TIM) family is associated with autoimmune diseases, but its expression level in the immune cells of systemic lupus erythematosus (SLE) patients is not known. The aim of this study was to investigate whether the expression of TIM-3 mRNA is associated with pathogenesis of SLE. Quantitative real-time reverse transcription-polymerase chain reaction analysis (qRT-PCR) was used to determine TIM-1, TIM-3, and TIM-4 mRNA expression in peripheral blood mononuclear cells (PBMCs) from 132 patients with SLE and 62 healthy controls. The PBMC surface protein expression of TIMs in PBMCs from 20 SLE patients and 15 healthy controls was assayed by flow cytometry. Only TIM-3 mRNA expression decreased significantly in SLE patients compared with healthy controls $(P<0.001)$. No significant differences in TIM family protein expression were observed in leukocytes from SLE patients and healthy controls $(P>0.05)$. SLE patients with lupus nephritis (LN) had a significantly lower expression of TIM-3 mRNA than those without $L N(P=0.001)$. There was no significant difference in the expression of TIM-3 mRNA within different classes of LN ( $P>0.05)$. Correlation of TIM-3 mRNA expression with serum IgA was highly significant $(r=0.425$, $P=0.004)$, but was weakly correlated with total serum protein $\left(r_{s}=0.283, P=0.049\right)$ and serum albumin $\left(r_{s}=0.297, P=0.047\right)$. TIM3 mRNA expression was weakly correlated with the Systemic Lupus Erythematosus Disease Activity Index $\left(\right.$ SLEDAl; $r_{s}=-0.272$, $\mathrm{P}=0.032$ ). Our results suggest that below-normal expression of TIM-3 mRNA in PBMC may be involved in the pathogenesis of SLE.
\end{abstract}

Key words: TIM-3; Systemic lupus erythematosus; Lupus nephritis; T-helper cell

\section{Introduction}

The T-cell immunoglobulin and mucin domain (TIM) family consists of three genes on human chromosome $5 q 33.2$, including the genes coding for TIM-1, TIM-3 and TIM-4 proteins (1). It has been reported that the TIM family is associated with organ-specific inflammatory and autoimmune diseases (2-4). TIM-1 is preferentially expressed on T-helper 2 (Th2) cells and is clinically significant as a human susceptibility gene for asthma, allergy, and autoimmunity. TIM-4 is exclusively expressed on antigenpresenting cells, where it mediates phagocytosis of apoptotic cells and has an important role in maintaining tolerance (1). In contrast, TIM-3 is a surface protein found on late-stage differentiated Th1 cells and inhibits aggressive Th1-mediated auto- and alloimmune responses (2).
There is increasing evidence that TIM-3 influences chronic autoimmune diseases. Galectin-9, a TIM-3 ligand, induces Th1 cell death in a TIM-3-dependent manner, and galectin-9-deficient mice become susceptible to collageninduced arthritis (CIA) $(5,6)$. Th17 cells and activated cytotoxic CD8 + T cells also express TIM-3 and are sensitive to galectin-9-induced apoptosis (6,7). In vivo administration of antibody to TIM-3 was reported to exacerbate the clinical and pathological severity of experimental autoimmune encephalomyelitis, a Th1/Th17-mediated autoimmune disease (8). The analysis of T-cell clones obtained from patients with multiple sclerosis revealed significantly lower expression of TIM-3 than seen in normal controls (9). Such findings indicate that TIM-3 may play an

Correspondence: Yi Jiang <xzcai@mail.cmu.edu.cn>

Received November 16, 2013. Accepted August 28, 2014. First published online October 17, 2014. 
important role in the development and pathogenesis of autoimmune diseases.

Systemic lupus erythematosus (SLE) is a prototypic autoimmune disease characterized by production of a diverse array of autoantibodies, complement activation, and deposition of immune complexes, causing tissue and organ damage. Most SLE patients develop kidney disease, and lupus nephritis (LN) is one of its most severe manifestations (10). It has been demonstrated that defects in the appropriate regulation of Th1 and Th17 cell function are implicated in the pathogenesis of SLE $(11,12)$. TIM-3 is preferentially expressed in Th1 and Th17 cells and may play a role in the development of SLE.

In this study, we looked for significant differences in the expression of TIM-1, TIM-3 and TIM-4 mRNA in peripheral blood mononuclear cells (PBMCs) from a relatively large population of SLE patients $(n=132)$ and healthy controls $(n=62)$. We found that in PBMCs from SLE patients the transcription level of only TIM-3 decreased significantly compared with healthy controls. Although TIM-1 and TIM-4 transcription increased in PBMCs from SLE patients, it did not reach statistical significance compared with healthy controls. Our results also showed that the expression of TIM-3 mRNA in SLE patients with LN was significantly lower than in those without LN. Moreover, the mRNA expression of TIM-3 correlated with several laboratory parameters, such as serum $\lg A$, serum total protein, and serum albumin. Therefore, our results indicated that TIM-3 may participate in the pathogenesis of SLE.

\section{Material and Methods}

\section{Human subjects}

One hundred and thirty-two patients with SLE diagnosed according to the criteria of the 1982 American College of Rheumatology were enrolled along with 62 healthy sex- and age-matched controls without autoimmune disease or cancer. Peripheral blood samples were collected from all controls and from all patients before they took any immunosuppressive drugs. Informed consent was obtained from all participants; the human ethics committee of China Medical University approved the study.

The demographic characteristics, clinical features, and laboratory measurements of SLE patients are reported in Table 1. The disease activity of the SLE patients was evaluated using the Systemic Lupus Erythematosus Disease Activity Index (SLEDAI). The SLEDAI score (mean \pm SE) of the patients with SLE was $15.8 \pm 8.1$. Kidney tissue biopsies were obtained for routine histological evaluation, immunofluorescence staining, and classification according to the International Society of Nephrology (ISN)/Renal Pathology Society (RPS) criteria (13).

\section{Preparation of PBMC and extraction of RNA}

PBMCs were separated by density gradient centrifugation $(3000 \mathrm{~g})$ from the peripheral blood anticoagulated
Table 1. Demographic characteristics, clinical features and laboratory measurements of systemic lupus erythematosus (SLE) patients.

\begin{tabular}{|c|c|}
\hline & $\begin{array}{l}\text { SLE patients } \\
(\mathrm{n}=132)\end{array}$ \\
\hline \multicolumn{2}{|l|}{ Demographic characteristics } \\
\hline Female, n (\%) & $112(85 \%)$ \\
\hline Male, n (\%) & $20(15 \%)$ \\
\hline Age (years) & $36 \pm 12$ \\
\hline \multicolumn{2}{|l|}{ Clinical features } \\
\hline Raynaud's phenomenon, n (\%) & $14(11 \%)$ \\
\hline Arthritis, n (\%) & $78(59 \%)$ \\
\hline Lupus nephritis, n (\%) & $52(40 \%)$ \\
\hline Serositis, n (\%) & $29(22 \%)$ \\
\hline CNS disease, $\mathrm{n}(\%)$ & $10(8 \%)$ \\
\hline SLEDAI & $15.8 \pm 8.1$ \\
\hline \multicolumn{2}{|l|}{ Laboratory measurements } \\
\hline ANA, n (\%) & $108(82 \%)$ \\
\hline Anti-dsDNA, n (\%) & $104(79 \%)$ \\
\hline Anti-Sm, n (\%) & $55(42 \%)$ \\
\hline C3 $(g / L)$ & $0.70 \pm 0.36$ \\
\hline C4 (g/L) & $0.15 \pm 0.10$ \\
\hline $\lg G(g / L)$ & $15.9 \pm 6.55$ \\
\hline $\lg M(g / L)$ & $1.29 \pm 0.73$ \\
\hline $\lg A(g / L)$ & $3.05 \pm 1.36$ \\
\hline $\operatorname{ESR}(\mathrm{mm} / \mathrm{h})$ & $32.8 \pm 20.06$ \\
\hline Serum total protein $(\mathrm{g} / \mathrm{L})$ & $61.6 \pm 16.02$ \\
\hline Serum albumin $(g / L)$ & $35.7 \pm 8.81$ \\
\hline Serum creatinine $(\mu \mathrm{mol} / \mathrm{L})$ & $61.7 \pm 33.76$ \\
\hline Serum CRP (mg/L) & $13.7 \pm 3.25$ \\
\hline Serum LDH $(\mathrm{U} / \mathrm{L})$ & $242.3 \pm 133.01$ \\
\hline 24-h proteinuria (g/day) & $10.44 \pm 5.78$ \\
\hline
\end{tabular}

Data are reported as means $\pm S D$, unless otherwise indicated. For the detection of ANA and anti-dsDNA autoantibodies, the cutoff value of the serum titer was 1:80. CNS: central nervous system; SLEDAl: Systemic Lupus Erythematosus Disease Activity Index; ANA: anti-nuclear antibody; dsDNA: double-stranded DNA; Sm: Smith; Ig: immunoglobulin; ESR: erythrocyte sedimentation rate; CRP: C-reactive protein; $\mathrm{LDH}$ : lactate dehydrogenase.

with sodium citrate, collected from the interphase and washed twice with phosphate-buffered saline. Total RNA was extracted using TRIzol (Invitrogen, USA) according to the manufacturer's protocol and then quantified by photometrical measurement.

\section{Quantitative real-time RT-PCR (qRT-PCR)}

One microgram of total RNA was reverse transcribed to cDNA in a total volume of $20 \mu \mathrm{L}$ using an $\mathrm{RT}$ reaction kit (Promega, USA). qRT-PCR was performed using the Express SYBR GreenER qPCR Supermix Universal Kit (Invitrogen) on a Rotor-gene 6000 system (Qiagen, Australia). The $25-\mu \mathrm{L}$ PCR mixture contained $2 \mu \mathrm{L}$ reversetranscribed product, $12.5 \mu \mathrm{L}$ SYBR Green Supermix, $8.5 \mu \mathrm{L}$ RNase-free water, and $1 \mu \mathrm{L}$ each of forward and reverse 
primers. The reaction was performed in a 72-well optical plate in triplicate. The first step of the PCR protocol was $95^{\circ} \mathrm{C}$ for $10 \mathrm{~s}$, followed by 40 cycles of $95^{\circ} \mathrm{C}$ for $5 \mathrm{~s}$, and $60^{\circ} \mathrm{C}$ for $30 \mathrm{~s}$ as the second step. A melting-curve analysis was performed to ensure specificity of the PCR products, and all the PCR products were separated by electrophoresis on agarose gels to isolate single bands of the expected size. The expression of TIMs was normalized to $\beta$-actin and determined using the comparative $2^{-\Delta \Delta C t}$ method (14). The primers used were: TIM-1 forward, 5'-GCTTTGCAAAATG CAGTTGA-3' and reverse, 5'-TGTTGGAATGCCAGATG AAA-3'; TIM-3 forward, 5'-GACTTCACTGCAGCCTT TCC-3' and reverse 5'-GATCCCTGCTCCGATGTAGA3'; TIM-4 forward, 5'-GGATTTGTGCTCTTCGCATT-3' and reverse, 5'-CCCCCATCСТCAАTCTAACA-3'; $\beta$-actin forward, 5'-TACAGCTTCACCACCACAGC-3' and reverse 5'-AAGGAAGGCTGGAAAAGAGC-3'; IFN- $\gamma$ forward, 5'-GCAGAGCCAAATTGTCTCCT-3' and reverse 5'-ATG CTCTTCGACCTCGAAAC-3'.

\section{Flow cytometric analysis}

Briefly, freshly collected whole-blood samples were incubated for $30 \mathrm{~min}$ at room temperature with fluorochrome-labeled monoclonal antibodies: TIM-1-PE, TIM-3FITC and TIM-4-PE (BD Biosciences, USA). The red blood cells were lysed for $15 \mathrm{~min}$ and, after washing twice with PBS, the remaining cells were assayed within $30 \mathrm{~min}$ by fluorescence-activated cell sorting (FACSCalibur, BD Biosciences). Flow cytometry data were collected and analyzed using the CellQuest software (BD Biosciences).

\section{Statistical analysis}

Data were evaluated using the SPSS 16.0 statistical software package (SPSS, USA). The relative mRNA expression observed in patients and controls are reported as group medians and ranges, and the significance of group differences was tested by the Mann-Whitney U-test. Correlations were calculated using Spearman's rank correlation coefficient $\left(r_{s}\right)$. All tests were two-tailed, and $P$ values $<0.05$ were considered to be statistically significant.

\section{Results}

Quantification of TIM-1, TIM-3, and TIM-4 expression in PBMCs from SLE patients and healthy controls

The expression of TIM-1, TIM-3 and TIM-4 mRNA in PBMCs in the 132 SLE patients and 62 healthy controls was assessed by qRT-PCR. Expression of TIM-3 mRNA in PBMCs from SLE patients [0.61 (0.0051-2.87)] was lower than that in controls $[1.13(0.19-3.98 ; \mathrm{P}<0.001)$ (Figure 1$)$. Expression of TIM-1 mRNA [0.61 (0.03-191.2) in controls vs $2.28(0.0066-255.8)$ in SLE patients, $P=0.131)]$ and of TIM4 [0.40 (0.063-69.5) in controls vs $0.92(0.018-29.5)$ in SLE patients, $P=0.233)$ ] were not significantly different between patients and controls. No significant differences in TIM-1, TIM-3 and TIM-4 leukocyte surface protein expression were

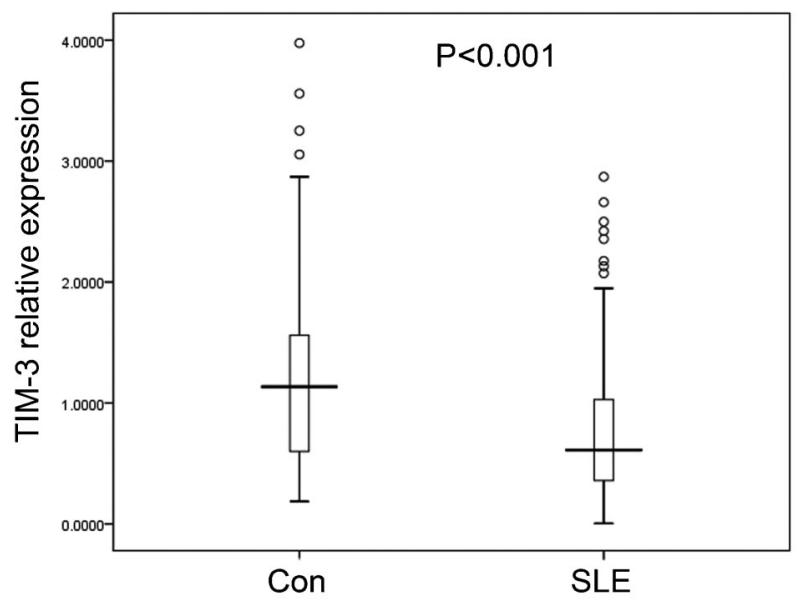

Figure 1. TIM-3 mRNA expression levels in peripheral blood mononuclear cells from systemic lupus erythematosus (SLE) patients $(n=132)$ and healthy controls (Con; $n=62)$. Data are reported in box plots as medians, quartiles, and outsiders (circles). $\mathrm{P}<0.001$, SLE patients compared to healthy controls (MannWhitney test).

observed in the 20 SLE patients and 15 healthy controls who were evaluated $(P>0.05)$.

\section{TIM-3 mRNA expression in PBMCs from SLE patients with and without LN}

The kidney biopsy tissues from SLE patients were evaluated for routine histology and immunofluorescence. LN was found in 52 of the 132 SLE patients (39.4\%) and was classified according to the ISN/RPS criteria. There were 8 patients (15\%) with class II, $5(10 \%)$ with class III, $29(55 \%)$ with class IV, 6 (12\%) with class V, and $4(8 \%)$ with class IV $+\mathrm{V}$ LN. The expression of TIM-3 mRNA in SLE patients with LN [0.49 (0.005-1.182)] was significantly lower than in those without LN [0.79 (0.042-2.871; $\mathrm{P}=0.001$; Figure 2]. At the same time, the expression of TIM-3 mRNA in patients within each class of LN was compared to determine whether there was any correlation of TIM-3 with the disease progression of LN. However, we found no significant differences in the expression of TIM-3 mRNA among the different classes of LN $(P>0.05)$.

\section{Analysis of relationships between TIM-3 mRNA expression and clinical characteristics, laboratory parameters, and Th1 cytokine in SLE patients}

Association of TIM-3 with laboratory parameters was analyzed. The mRNA expression of TIM-3 was significantly correlated with serum $\lg A\left(r_{s}=0.425, P=0.004\right)$, and weakly correlated with serum total protein and serum albumin $\left(r_{s}=0.283, P=0.049 ; r_{s}=0.297, P=0.047\right.$, respectively). A weak, negative correlation between TIM-3 mRNA expression and SLEDAI was also found $\left(r_{s}=-0.272, P=\right.$ 0.032 ). No significant correlations were observed between TIM-3 mRNA expression and other characteristics or 


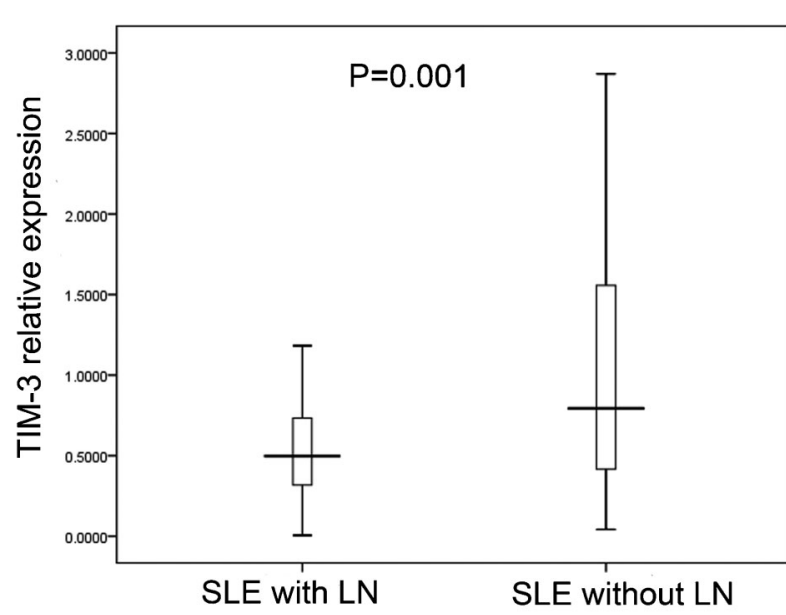

Figure 2. TIM-3 mRNA expression levels in peripheral blood mononuclear cells from systemic lupus erythematosus (SLE) patients with and without lupus nephritis (LN). Data are reported in box plots as medians and quartiles. $P=0.001$, SLE patients with $\mathrm{LN}(\mathrm{n}=52)$ compared to SLE patients without $\mathrm{LN}(\mathrm{n}=80)$ (Mann-Whitney test).

laboratory parameters in SLE patients (Table 2). As dysregulated cytokines may be linked to the pathogenesis of SLE, we assayed the expression of Th1-type cytokine IFN- $\gamma$ mRNA and investigated the correlation between the expression of IFN- $\gamma$ and TIM-3 in PBMC from SLE patients. The expression of IFN- $\gamma$ mRNA was significantly greater in the 132 SLE patients [0.42 $(0.127-22.061]$ than in the 62 healthy controls [0.30 (0.060-1.639); $P=0.011]$. No significant correlation was observed between the mRNA expression of $\mathrm{TIM}-3$ and IFN- $\gamma$ in the SLE patients participating in our study.

Table 2. Correlation between TIM-3 mRNA expression and clinical parameters in systemic lupus erythematosus (SLE) patients.

\begin{tabular}{lrc}
\hline SLE patients & \multicolumn{1}{c}{$\mathrm{r}_{\mathrm{s}}$} & $\mathrm{P}$ \\
\hline $\mathrm{C} 3$ & 0.154 & 0.318 \\
$\mathrm{C} 4$ & -0.107 & 0.488 \\
$\mathrm{IgG}$ & 0.256 & 0.094 \\
$\mathrm{IgM}$ & 0.154 & 0.320 \\
IgA & 0.425 & 0.004 \\
ESR & -0.143 & 0.449 \\
Serum total protein & 0.283 & 0.049 \\
Serum albumin & 0.297 & 0.047 \\
Serum creatinine & -0.114 & 0.460 \\
Serum CRP & -0.143 & 0.378 \\
Serum LDH & -0.241 & 0.151 \\
24-h proteinuria & 0.073 & 0.683 \\
SLEDAl & -0.272 & 0.032 \\
\hline
\end{tabular}

Spearman's test was used for statistical analyses. See Table 1 for abbreviations.

\section{Discussion}

Naive T-helper cells chiefly differentiate into Th1 or Th2 cells, and defects in the appropriate regulation of their function have been implicated in the pathophysiology of SLE. Recent studies have indicated that inappropriate regulation of Th17 cells also participates in the pathogenesis of SLE $(12,15)$. The TIM family represents a group of molecules that, in concert with T-cell receptors and costimulatory signals, regulate the expansion and effector functions of Th cells $(16,17)$. In this study, we explored the mRNA expression of TIM-1, TIM-3 and TIM-4 in PBMCs from SLE patients and healthy controls using qRT-PCR. We found that the mRNA expression of TIM-3 decreased significantly in PBMCs from SLE patients. Unfortunately, although the mRNA expression of TIM-1 and TIM-4 in PBMCs from SLE patients showed an increase, it did not reach statistical significance compared with healthy controls.

Since transcript levels do not always correlate with protein levels, we assayed the expression of TIM-1, -3 and -4 surface proteins by flow cytometry in a limited number of patients and controls. No significant differences in expression of TIM surface proteins were observed in the 20 SLE patients and 15 healthy controls who were evaluated. Estimation of serum or leukocyte-associated TIM-3 expression in larger study populations is needed for further validation of the data. Wang et al. (18) reported that TIM-1 expression increased significantly in 19 SLE patients compared with that in 19 healthy controls, but that the decrease in TIM-3 expression was not statistically significant. The contradictory results may be due to differences in sample size, variations in case source, or the effects of a poorly understood variable that itself needs further study.

In the MRL/pr mouse model of lupus, investigators have reported that a cytokine imbalance with Th1 predominance was associated with acceleration of a lupus-like autoimmune syndrome, and elevated expression of the IF $N-\gamma$ gene was correlated with severity of the disease $(19,20)$. Therefore, we assessed the mRNA expression of the Th1-type cytokine IFN- $\gamma$ in PBMC from SLE patients and healthy controls. We found that IFN- $\gamma$ mRNA expression in the SLE patients was significantly higher than in the healthy controls. This result confirms the previous reports of Csiszar et al. (21) and Harigai et al. (22). However, our study failed to show any correlation between IFN- $\gamma$ and TIM-3. We speculate that although TIM-3 and IFN- $\gamma$ play important roles in Th1 cells and in the pathogenesis of SLE, they may be regulated in different ways.

Our study also showed that the expression of TIM-3 mRNA was significantly lower in SLE patients with LN than in those without LN. However, our results showed that there were no significant differences in the expression of TIM-3 mRNA among the different classes of LN. Considering the small number of participants in each LN class, future 
studies should collect and classify more kidney biopsy tissue samples.

$\mathrm{LN}$ is a common and serious complication of SLE patients, and is considered to be the crucial factor of prognosis in SLE (23). We found in previous studies that TIM-3 negatively regulated autoimmunity or inflammatory diseases, including multiple sclerosis and neuritis, as well as nephritis $(9,24,25)$. TIM-3 is preferentially expressed on Th1 cells vs Th2 cells and known to negatively regulate Th1 Tcell responses. TIM-3 may also be able to affect Th2-driven disease by indirectly modulating the balance between Th1and Th2-type responses. Skewing of the Th1/Th2 balance toward Th2 by TIM-3 blockade may contribute to the renal damage of SLE (26). In addition, the TIM-3 ligand could ameliorate proteinuria and suppress anti-dsDNA antibody production by regulating T-cell function, although TIM-3 may not be directly involved in the process (27). The fact that TIM-3 ligand reduces the numbers of TIM- $3^{+} \mathrm{CD} 8^{+} \mathrm{T}$ cells in MRL/lpr mice suggests that $\mathrm{TIM}-3^{+} \mathrm{CD} 8^{+} \mathrm{T}$ cells are associated with lupus pathogenesis, since infiltration of $\mathrm{CD}^{+} \mathrm{T}$ cells in lupus kidney indicates that they have the potential to mediate kidney injury $(27,28)$. It has also been reported that TIM-3 negatively regulates the activation of macrophages in the kidney during nephrotoxic serum nephritis (NTS), and that TIM-3 blockade aggravates NTS by increasing the number of kidney-infiltrating inflammatory cells. Both effects provide evidence that TIM-3 exerts a protective influence in the course of nephritis (25). Therefore, it seems that stimulation of TIM-3 expression may be a promising target for therapeutic intervention in autoimmune nephritis.

To gain insight into the relation of TIM-3 with diseaseassociated parameters, we determined their levels in SLE patients and evaluated their correlation with TIM-3 mRNA expression. We found that the expression of TIM-3 mRNA was significantly correlated with $\lg \mathrm{A}$, and weakly correlated with serum total protein, serum albumin, and SLEDAI because of the marginal $P$ values. It is really difficult to determine the precise mechanisms of the correlation between TIM-3 expression and total protein

\section{References}

1. Freeman GJ, Casasnovas JM, Umetsu DT, DeKruyff RH. TIM genes: a family of cell surface phosphatidylserine receptors that regulate innate and adaptive immunity. Immunol Rev 2010; 235: 172-189.

2. Sanchez-Fueyo A, Tian J, Picarella D, Domenig C, Zheng $X X$, Sabatos $C A$, et al. Tim-3 inhibits $T$ helper type 1mediated auto- and alloimmune responses and promotes immunological tolerance. Nat Immunol 2003; 4: 1093-1101, doi: 10.1038/ni987.

3. McIntire JJ, Umetsu SE, Akbari O, Potter M, Kuchroo VK, Barsh GS, et al. Identification of Tapr (an airway hyperreactivity regulatory locus) and the linked Tim gene family. Nat Immunol 2001; 2: 1109-1116, doi: 10.1038/ni739. and albumin. The correlation may result from autoimmunity and disease development, and may be involved in the severity of SLE disease. The insufficient expression of TIM-3 may cause hyperactivation of autoreactive T cells, which is one of the hallmarks of SLE. TIM-3 is selectively expressed on fully differentiated Th1, and is a key regulator of self-reactive Th1 cells and maintenance of tolerance in autoimmune disease $(2,9)$. Its decreased expression may induce hyperactivation of $\mathrm{T}$ cells and the subsequent organ damage in SLE patients, such as the kidney. Impairment of renal function may result in abnormal levels of serum total protein and albumin. In addition, our results showed that there was a significant difference in TIM-3 expression between SLE patients with LN and those without LN. The association of TIM-3 expression with LN and laboratory parameters in SLE patients also indicated that reduction of TIM-3 expression may be associated with the severity of SLE disease. Taken together, analysis of the relationship between TIM3 mRNA expression and SLE-IgA, serum total protein, serum albumin, and SLEDAI further suggested a potential role of decreased TIM-3 mRNA expression in the pathogenesis of SLE.

In summary, we have demonstrated that TIM-3 mRNA expression is lower in SLE patients than in healthy controls. We also showed that TIM-3 mRNA expression in SLE patients with LN was significantly lower than in those without LN. Moreover, the expression of TIM-3 mRNA correlated with levels of serum IgA, total protein, and albumin, and was weakly inversely correlated with SLEDAI. The evidence indicated that TIM-3 may play a part in the pathogenesis of SLE and may be a promising target for therapeutic intervention in SLE.

\section{Acknowledgments}

We especially thank all SLE patients and colleagues who participated for making this study possible. Research supported by the National Nature Science Foundation of China (\#30571701, \#30600541).

4. Khademi M, Illes Z, Gielen AW, Marta M, Takazawa N, Baecher-Allan C, et al. T Cell Ig- and mucin-domain-containing molecule-3 (TIM-3) and TIM-1 molecules are differentially expressed on human Th1 and Th2 cells and in cerebrospinal fluid-derived mononuclear cells in multiple sclerosis. J Immunol 2004; 172: 7169-7176, doi: 10.4049/jimmunol.172. 11.7169.

5. Zhu C, Anderson AC, Schubart A, Xiong H, Imitola J, Khoury SJ, et al. The Tim-3 ligand galectin-9 negatively regulates T helper type 1 immunity. Nat Immunol 2005; 6: 1245-1252, doi: 10.1038/ni1271.

6. Seki M, Oomizu S, Sakata KM, Sakata A, Arikawa T, Watanabe K, et al. Galectin-9 suppresses the generation of 
Th17, promotes the induction of regulatory $\mathrm{T}$ cells, and regulates experimental autoimmune arthritis. Clin Immunol 2008; 127: 78-88, doi: 10.1016/j.clim.2008.01.006.

7. Wang F, He W, Zhou H, Yuan J, Wu K, Xu L, et al. The Tim3 ligand galectin- 9 negatively regulates CD8 + alloreactive T cell and prolongs survival of skin graft. Cell Immunol 2007; 250: 68-74, doi: 10.1016/j.cellimm.2008.01.006.

8. Monney L, Sabatos CA, Gaglia JL, Ryu A, Waldner H, Chernova $\mathrm{T}$, et al. Th1-specific cell surface protein Tim-3 regulates macrophage activation and severity of an autoimmune disease. Nature 2002; 415: 536-541, doi: 10.1038/ 415536a.

9. Koguchi K, Anderson DE, Yang L, O'Connor KC, Kuchroo VK, Hafler DA. Dysregulated T cell expression of TIM3 in multiple sclerosis. J Exp Med 2006; 203: 1413-1418.

10. Giannico G, Fogo AB. Lupus nephritis: is the kidney biopsy currently necessary in the management of lupus nephritis? Clin J Am Soc Nephrol 2013; 8: 138-145, doi: 10.2215/CJN. 03400412.

11. Charles N, Hardwick D, Daugas E, Illei GG, Rivera J. Basophils and the T helper 2 environment can promote the development of lupus nephritis. Nat Med 2010; 16: 701-707, doi: 10.1038/nm.2159.

12. Pan HF, Zhang N, Li WX, Tao JH, Ye DQ. TIM-3 as a new therapeutic target in systemic lupus erythematosus. Mol Biol Rep 2010; 37: 395-398, doi: 10.1007/s11033-009-9833-7.

13. Weening JJ, D'Agati VD, Schwartz MM, Seshan SV, Alpers CE, Appel GB, et al. The classification of glomerulonephritis in systemic lupus erythematosus revisited. J Am Soc Nephrol 2004; 15: 241-250, doi: 10.1097/01.ASN.0000108969.21691. $5 \mathrm{D}$.

14. Livak KJ, Schmittgen TD. Analysis of relative gene expression data using real-time quantitative PCR and the 2(-Delta Delta C(T)) Method. Methods 2001; 25: 402-408, doi: $10.1006 /$ meth.2001.1262.

15. Yuan J, Yu M, Cao AL, Chen X, Zhang LH, Song Y, et al. A novel epitope from CD22 regulates Th1 and Th17 cell function in systemic lupus erythematosus. PLoS One 2013; 8: e64572, doi: 10.1371/journal.pone.0064572.

16. Mariat C, Sanchez-Fueyo A, Alexopoulos SP, Kenny J, Strom TB, Zheng XX. Regulation of $T$ cell dependent immune responses by TIM family members. Philos Trans $R$ Soc Lond B Biol Sci 2005; 360: 1681-1685, doi: 10.1098/ rstb.2005.1706

17. Su EW, Lin JY, Kane LP. TIM-1 and TIM-3 proteins in immune regulation. Cytokine 2008; 44: 9-13, doi: 10.1016/j.cyto.2008. 06.013.

18. Wang Y, Meng J, Wang X, Liu S, Shu Q, Gao L, et al.
Expression of human TIM-1 and TIM-3 on lymphocytes from systemic lupus erythematosus patients. Scand J Immunol 2008; 67: 63-70.

19. Manolios N, Schrieber L, Nelson M, Geczy CL. Enhanced interferon-gamma (IFN) production by lymph node cells from autoimmune (MRL/1, MRL/n) mice. Clin Exp Immunol 1989; 76: 301-306.

20. Takahashi S, Fossati L, Iwamoto M, Merino R, Motta R, Kobayakawa T, et al. Imbalance towards Th1 predominance is associated with acceleration of lupus-like autoimmune syndrome in MRL mice. J Clin Invest 1996; 97: 1597-1604, doi: $10.1172 / \mathrm{JCl} 118584$.

21. Csiszar A, Nagy G, Gergely P, Pozsonyi T, Pocsik E. Increased interferon-gamma (IFN-gamma), IL-10 and decreased IL-4 mRNA expression in peripheral blood mononuclear cells (PBMC) from patients with systemic lupus erythematosus (SLE). Clin Exp Immunol 2000; 122: 464-470, doi: 10.1046/j.1365-2249.2000.01369.x.

22. Harigai M, Kawamoto M, Hara M, Kubota T, Kamatani N, Miyasaka N. Excessive production of IFN-gamma in patients with systemic lupus erythematosus and its contribution to induction of B lymphocyte stimulator/B cell-activating factor/ TNF ligand superfamily-13B. J Immunol 2008; 181: 22112219, doi: 10.4049/jimmunol.181.3.2211.

23. Liu ZC, Zhou QL. Tumor necrosis factor-like weak inducer of apoptosis and its potential roles in lupus nephritis. Inflamm Res 2012; 61: 277-284, doi: 10.1007/s00011-011-0420-8.

24. Zhang ZY, Schluesener HJ, Zhang Z. Distinct expression of Tim-3 during different stages of rat experimental autoimmune neuritis. Brain Res Bull 2011; 86: 229-234, doi: 10.1016/j.brainresbull.2011.07.005.

25. Schroll A, Eller K, Huber JM, Theurl IM, Wolf AM, Weiss G, et al. Tim3 is upregulated and protective in nephrotoxic serum nephritis. Am J Pathol 2010; 176: 1716-1724.

26. Lee J, Phong B, Egloff AM, Kane LP. TIM polymorphisms genetics and function. Genes Immun 2011; 12: 595-604, doi: 10.1038/gene.2011.75.

27. Moritoki M, Kadowaki T, Niki T, Nakano D, Soma G, Mori H, et al. Galectin-9 ameliorates clinical severity of MRL/lpr lupus-prone mice by inducing plasma cell apoptosis independently of Tim-3. PLoS One 2013; 8: e60807, doi: 10.1371/journal.pone.0060807.

28. Winchester R, Wiesendanger M, Zhang HZ, Steshenko V, Peterson K, Geraldino-Pardilla L, et al. Immunologic characteristics of intrarenal T cells: trafficking of expanded CD8 + $\mathrm{T}$ cell beta-chain clonotypes in progressive lupus nephritis. Arthritis Rheum 2012; 64: 1589-1600, doi: 10.1002/art. 33488. 